\title{
Análisis florístico de la selva tropical perennifolia del centro-norte de Veracruz, México \\ Floristic analysis of the tropical evergreen forest in the center-north of Veracruz, Mexico
}

\author{
Mireya Burgos-Hernández ${ }^{1,2 *}$, Gonzalo Castillo-Campos ${ }^{1}$ \\ ${ }^{1}$ Red de Biodiversidad y Sistemática. Instituto de Ecología A.C. Carretera antigua a Coatepec 351, EI Haya. Xalapa, CP. \\ 91070. Veracruz, México. \\ ${ }^{2}$ Departamento de Botánica. Colegio de Postgraduados. Carretera México-Texcoco Km 36.5, Montecillo 53230. Texcoco, \\ Estado de México, México. \\ ${ }^{*}$ Autor de correspondencia: mireya_bh14@hotmail.com
}

Artículo científico recibido: 08 de noviembre de 2017 aceptado: 14 de marzo de 2018

RESUMEN. El objetivo fue caracterizar y describir la composición florística de la selva tropical perennifolia del centronorte del estado de Veracruz, México. Se realizó un inventario florístico por el método de parcelas. Para identificar la heterogeneidad entre parcelas de muestreo, se efectuó un análisis de similitud de Jaccard y un análisis de componentes principales (ACP). El estado de conservación se analizó con los criterios de especies registradas, frecuencia, coberturaabundancia, formas de crecimiento, estructura vertical y especies amenazadas. La lista florística incluye 180 especies, 129 géneros y 74 familias. Se detectaron siete especies en categoría de riesgo y una endémica del estado. El análisis de similitud de Jaccard y el ACP determinaron diferencias florísticas entre las parcelas muestreadas. El fragmento de selva estudiado se encuentra conservado y resguarda una gran riqueza de especies nativas. Las características inherentes a la selva, su alta riqueza de especies, la presencia de especies endémicas y en riesgo, así como la pequeña superficie que ocupa, indica que se deben de establecer medidas para su conservación. Se sugiere que el remanente de selva estudiado se integre en las estrategias de conservación de los trópicos del país, con base en la riqueza de especies que salvaguarda.

Palabras clave: Atzalan, conservación, flora vascular, riqueza florística, selva alta perennifolia

ABSTRACT. The objective was to characterize and describe the floristic composition of the tropical evergreen forest in the center-north of the state of Veracruz, Mexico. A floristic inventory was carried out by the parcel method. To identify the heterogeneity among sampling plots, a Jaccard similarity analysis and a principal component analysis (PCA) were performed. The state of conservation was analyzed with the criteria of recorded species, frequency, coverageabundance, growth forms, vertical structure and threatened species. The floristic list includes 180 species, 129 genera and 74 families. Seven species were detected in the risk category and one endemic to the state. The Jaccard similarity analysis and PCA determined floristic differences among the plots sampled. The forest fragment studied is conserved and protects a great richness of native species. The inherent characteristics of the forest, its high species richness and the presence of endemic and at-risk species, as well as the small area it occupies, indicate that measures must be established for its conservation. It is suggested that the forest remnant studied be integrated into the country's tropical conservation strategies, based on the species richness that it safeguards.

Key words: Atzalan, conservation, vascular flora, floristic richness, evergreen forest

\section{INTRODUCCIÓN}

Los paisajes tropicales del mundo están cambiando de forma rápida, debido a la ocupación del territorio, el acelerado crecimiento poblacional, y las demandas socioeconómicas que propician de forma directa o indirecta incremento en la tasa de deforestación (Díaz-Gallegos et al. 2010, Ezcurra 2016). En México, las selvas tropicales del sureste son parte de la cubierta forestal que se ex- 


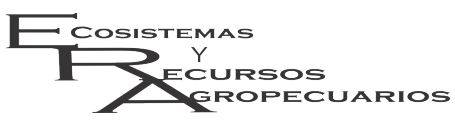

Burgos-Hernández y Castillo-Campos Análisis florístico de la selva tropical Ecosist. Recur. Agropec. 5(15):451-463,2018

tiende hasta el Petén en Guatemala y al noreste de Belice, formando parte del corredor biológico mesoamericano. En 1990, era considerado área prioritaria del flujo genético y la viabilidad de la biodiversidad tropical de Centro América (Miller et al. 2001). Pero aún con su importancia en la conectividad biológica, la deforestación le ha ganado terreno (Sánchez-Colón et al. 2009). Se estima que México contaba originalmente con cerca de 25 millones de hectáreas de selvas tropicales húmedas, cifra que en 1970 se redujo a 7.9 millones de hectáreas, presentando los cambios más drásticos los estados de Veracruz y Tabasco (Sánchez-Colón et al. 2009). Lo que coincide con las estimaciones realizadas por Villafuerte et al. (1993), quienes establecen que la mayor tasa de deforestación ocurrió entre 1969 y 1970. Estimaciones realizadas por Challenger et al. (2009) reportan que sólo persiste el $17.5 \%$ de la vegetación primaria del trópico húmedo de México; mientras que el FRA (2010) sólo reporta el 4.6\%.

En la actualidad, los remanentes de mayor extensión de selva tropical húmeda se encuentran ubicados en la región Lacandona del estado de Chiapas, los Chimalapas en Oaxaca, Los Tuxtlas en Veracruz y Calakmul en la Península de Yucatán, con fragmentos de menor tamaño, dispersos y aislados (Díaz-Gallegos et al. 2010). Particularmente la selva de Los Tuxtlas, es considerada el remanente más septentrional del continente americano (Guevara et al. 2000), y por lo tanto el límite actual norte de las selvas húmedas en este continente (Martínez-Ramos et al. 2016). Por lo que la mayoría de los esfuerzos de muestreo y conservación se han enfocado en esta selva (Zambrano et al. 2014); siendo Los Tuxtlas una de las regiones donde mejor se ha documentado la deforestación y los procesos de fragmentación de la selva tropical (Castillo-Campos y Laborde-Dovalí 2004), a tal magnitud, que la cobertura vegetal en la reserva se ha reducido a 640 de las casi 26500 ha de vegetación original (Martínez-Ramos et al. 2016). La pérdida acelerada de selvas tropicales húmedas ha ocasionado la necesidad de identificar áreas remanentes con alta riqueza de especies y, aunque los efectos de la pérdida y fragmentación del hábitat sobre la biodiversidad han sido bien documentados (Fahrig 2003), pocos estudios se han centrado en la estructura y composición florística de fragmentos de menor tamaño, que juegan un papel importante en la biodiversidad (Arroyo-Rodríguez et al. 2008).

El estado de Veracruz es uno de los tres estados con mayor diversidad florística del país, y forma parte de una de las floras mejor estudiadas de México (Rzedowski 1993). Resalta la zona central del estado, que representa uno de los cinco refugios primarios del Pleistoceno, donde se encuentra alta diversidad y endemismos de flora vascular (CastilloCampos et al. 2005). A pesar de los esfuerzos para tener representada la riqueza y diversidad florística de los ecosistemas tropicales, aún hay regiones que permanecen poco conocidas, como la flora que se encuentra dentro del polígono del municipio de Atzalan, en el centro-norte del estado de Veracruz. Para contribuir al conocimiento de la flora de las regiones tropicales de México, y brindar bases útiles para las estrategias de conservación y manejo de la selva tropical húmeda americana, el presente estudio tuvo el objetivo de caracterizar y describir la composición florística de la selva alta perennifolia del centro-norte del estado de Veracruz.

\section{MATERIALES Y MÉTODOS}

\section{Área de estudio}

El municipio de Atzalan se localiza en la región centro-norte del estado de Veracruz, en la zona montañosa de la sierra de Chiconquiaco, entre las coordenadas geográficas de los $19^{\circ} 52^{\prime} 20^{\prime \prime}$ a $19^{\circ}$ 53' $20^{\prime \prime}$ LN y $97^{\circ} 02^{\prime} 15^{\prime \prime}$ a $97^{\circ} 03^{\prime} 08^{\prime \prime}$ LO (Figura 1). Su altitud muestra una marcada variación, que va de los 56 a $1799 \mathrm{msnm}$, por lo que es posible encontrar diversos tipos de vegetación (Gobierno del estado de Veracruz 2017).

El estudio se realizó en uno de los últimos remanentes de selva alta perennifolia más septentrionales del continente Americano, con superficie aproximada de 45 ha. Los sitios de colecta se ubican entre las localidades de San Pedro Altepepan, La Vega del río San Pedro, Plan de la Luz y El Quimite, a una altitud de entre 600 y 800 msnm. 
(a)

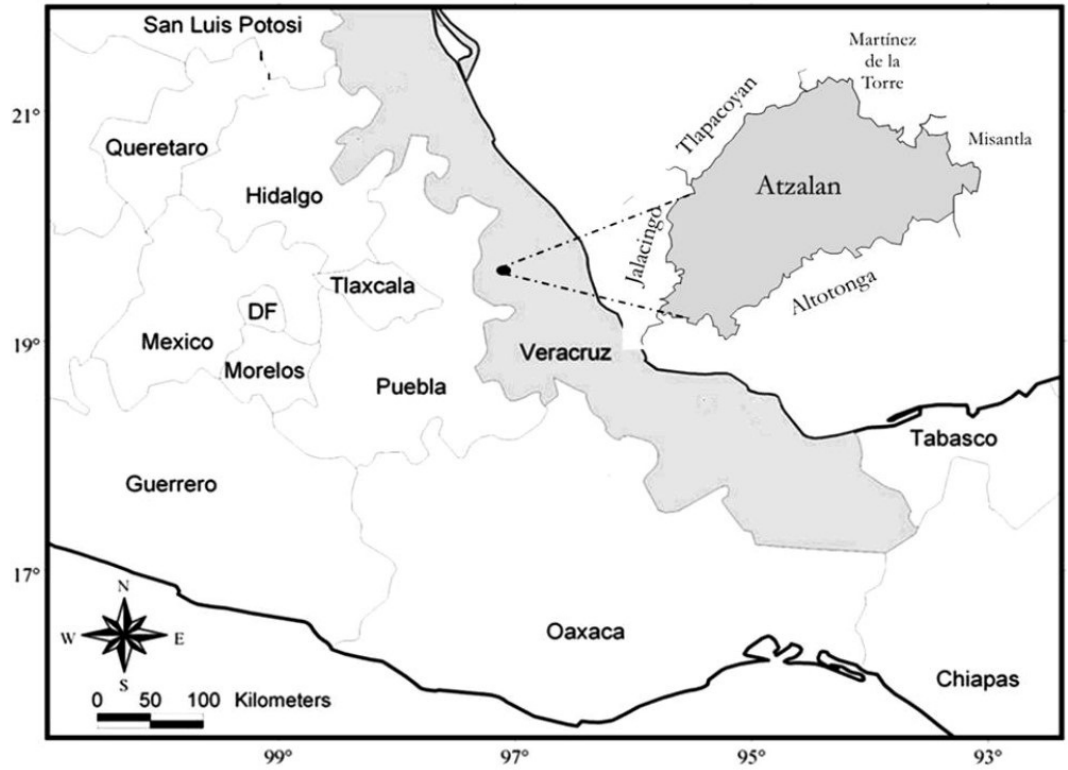

(b)

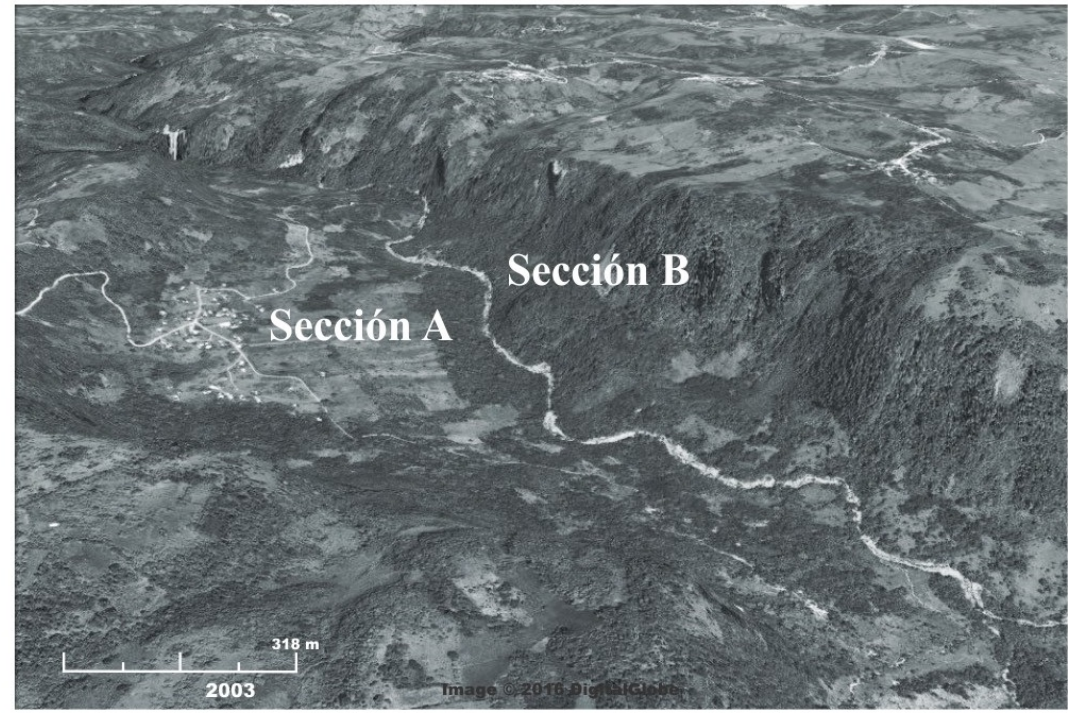

Figura 1. a) Localización del municipio de Atzalan en la región centro-norte del estado de Veracruz, México; b) Sitio de estudio.

El fragmento se encuentra dividido en dos secciones debido al paso del río San Pedro, una de ellas más cercana al poblado de La Vega (A) y la otra, al de San Pedro Altepepan (B).

\section{Muestreo florístico}

Para el muestreo de la vegetación se establecieron de manera aleatoria 24 parcelas de 100 $\mathrm{m}^{2}(10 \times 10 \mathrm{~m})$ para inventariar el estrato arbóreo y arbustivo, 12 en cada sección de selva. Dentro de cada parcela se establecieron al azar, tres sub-parce- 


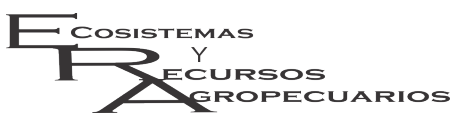

Burgos-Hernández y Castillo-Campos Análisis florístico de la selva tropical Ecosist. Recur. Agropec. 5(15):451-463,2018

las de $4 \mathrm{~m}^{2}$ para inventariar la vegetación herbácea (Castillo-Campos et al. 2008). En total se muestreó una superficie de $2400 \mathrm{~m}^{2}$. La vegetación se caracterizó considerando diferentes variables físicas y ambientales por parcela de muestreo, como la pendiente, orientación, cobertura de leñosas, cobertura de herbáceas, porcentaje de suelo desnudo, porcentaje de pedregosidad, altitud y porcentaje de cobertura vegetal. Para medir la cobertura se utilizó la escala de cobertura abundancia de BraunBlanquet modificada por van der Maarel (1979), que incluye las siguientes categorías de acuerdo al porcentaje de cobertura: 1 (1-3 individuos y menos del $5 \%$ de cobertura), 2 (3-10 individuos y menos del 5\%), 3 (más de 10 individuos y menos del 5\%), 4 (menos del 5\% y poco abundante), 5 (5-12.5\%), 6 (12.6-25\%), 7 (25.1-50\%), 8 (50.1-75\%), y 9 (75.1$100 \%)$.

El material florístico recabado se procesó y deposito en la colección del herbario (XAL) del Instituto de Ecología. Los ejemplares se identificaron con bibliografía especializada, las claves taxonómicas, los listados florísticos de la Flora de Veracruz (Gentry 1996, Sánchez-Vindas 1990, Vovides 1994, Mickel y Smith 2004, Acebey y Kömer 2008) y por comparaciones con material de herbario previamente identificado por expertos. Los nombres taxonómicos se corroboraron con la base de datos de The International Plant Name Index (IPNI 2017). El arreglo del listado florístico se realizó de acuerdo con Angiosperm Phylogeny Groups (APG IV 2016). Mientras que para los helechos y afines se uso la nomenclatura de Christenhusz et al. (2011).

El esfuerzo de muestreo se evaluó con una curva de acumulación de especies, con el paquete estadístico EstimateS 9.1.0 (Colwell 2013). Para ello, se empleó el estimador Chao 2, el cual fue elegido por presentar menor sesgo, además de que está basado en incidencias (presencia-ausencia) (LópezGómez y Williams-Linera 2006). Adicionalmente, se determinó la proporción de la riqueza de especies inventariada $(t q)$ de acuerdo al esfuerzo de muestreo a partir de la ecuación del modelo de Clench (Guevara y Dirzo 1998): $t q=\frac{q}{[b(1-q)]}$. Dónde: $q=\frac{S n}{\frac{a}{b}}$, $\mathrm{a}=$ tasa de crecimiento de nuevas especies, $\mathrm{b}$ = parámetro relacionado con la forma de la curva (Jiménez-Valverde y Hortal 2003), Sn = probabilidad de encontrar una especie.

Para considerar un muestreo representativo, el cálculo de la porción inventariada del total de las especies en una zona debe ser superior al $70 \%$ (Colwell 2013). Con base a lo anterior, se consideró para este estudio una proporción igual o superior al $80 \%(t q=0.8)$. Dicho análisis se llevó a cabo usando el paquete vegan (Oksanen et al. 2009) del programa estadístico $R$ versión 3.3.1.

\section{Estado de conservación del fragmento de selva}

El estado de conservación del fragmento de selva se evaluó con los criterios de frecuencias de las especies registradas, cobertura-abundancia, formas de crecimiento más frecuente y observación directa de la estructura vertical en los sitios de muestreo. También se consideró la estructura florística, usando como referencia la descripción de las especies más citadas y comúnmente consideradas como estructurales de una selva conservada de acuerdo con Gómez-Pompa (1966), Rzedowski (1978), Pennington y Sarukhán (2005), y Levy-Tacher et al. (2006). Adicionalmente, se consideraron las especies en categoría de riesgo, para lo cual se consultó la Norma Oficial Mexicana NOM059-SERMANAT-2010 y la Lista Roja de Especies Amenazadas de la Unión Internacional para Conservación de la Naturaleza (IUCN 2016).

Con base en el listado florístico de las 24 parcelas, se elaboró una matriz binaria de presenciaausencia para evaluar la similitud/disimilitud en la composición de especies inventariada, empleando el coeficiente de Jaccard (Moreno 2001). La matriz resultante se utilizó para construir un dendrograma con el método de la media aritmética no ponderada (UPGMA). Para conocer los patrones gráficos de la variación florística entre las parcelas de muestreo, se realizó un análisis de componentes principales (ACP) sobre la matriz de la covarianza de los datos de las especies. Los análisis se realizaron con el programa SYSTAT versión 13.1 (Systat 2016). 


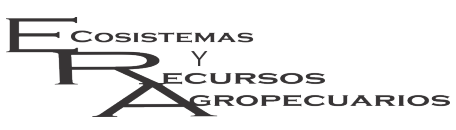

Burgos-Hernández y Castillo-Campos Análisis florístico de la selva tropical Ecosist. Recur. Agropec. 5(15):451-463,2018

\section{RESULTADOS}

\section{Inventario florístico}

Se muestreó una superficie de $2400 \mathrm{~m}^{2}$ de un remanente de selva alta perennifolia en la región centro-norte del estado de Veracruz. De acuerdo con la curva de acumulación de especies el muestreo fue suficiente (Figura 2), lo que se corroboró con la fórmula de proporción inventariada del modelo de Clench. Los resultados indican que el muestreo representa el $80 \%$ de la flora total $(t q=0.8=24$ parcelas de $100 \mathrm{~m}^{2}$ ), con probabilidad de 0.01 de aumentar un nuevo registro, solo si 28 parcelas más son muestreadas $(K=28.74)$.

Se registraron 180 especies de plantas, pertenecientes a 129 géneros y 74 familias. El 75\% de las especies inventariadas constituyen nuevos registros para el municipio. Las familias con mayor número de taxa fueron Araceae, Piperaceae y Rubiaceae, que en conjunto aportan $22 \%$ de las especies registradas. Las familias con el mayor número de géneros fueron Rubiaceae con seis, Malvaceae, Euphorbiaceae y Araceae con cinco cada una. En conjunto, estas familias aportan el $16 \%$ de los géneros registrados, de los cuales Piper, Philodendron y Psychotria presentaron la mayor riqueza, con nueve especies para el primero y seis para los dos últimos (Tabla 1). Las especies más frecuentes en los sitios de muestreo fueron: Beilschmiedia anay (S.F.Blake) Kosterm., Calatola mollis Standl., Philodendron seguine Schott, Oecopetalum mexicanum Greenm. \& C.H.Thomps., Anthurium flexile Schott, Syngonium podophyllum Schott, Ardisia compressa Kunth, Guarea glabra Vahl, Trophis mexicana (Liebm.) Boreau, Miconia trinervia D.Don ex Loudon y Miconia appendiculata Triana. La forma de crecimiento herbácea fue la mejor representada (36\%), seguida por la arbórea $(29 \%)$, epífitas y bejucos (18\%) y en menor proporción, el crecimiento arbustivo (17\%).

\section{Caracterización de la selva}

El fragmento de selva tropical perennifolia en el municipio de Atzalan se distribuye en una zona de difícil acceso, rodeado por zonas agrícolas y pas- tizales. Se ubica entre los 600 y $800 \mathrm{msnm}$, entre las localidades de San Pedro Altepepan y la Vega del río San Pedro. Es un tipo de vegetación característico de zonas tropicales de baja altitud, compuesta y dominada por varias especies de árboles tropicales. Presenta una cobertura de leñosas de aproximadamente $90 \%$, sin suelo desnudo y con una mínima presencia de tocones $(2 \%)$, sin pedregosidad y sin aparente perturbación. Se desarrolla entre cañadas y laderas con pendientes pronunciadas sobre un suelo arcilloso-arenoso, que de acuerdo a sus características, es rico en materia orgánica en los horizontes superiores. Presenta una estructura vertical compleja y multiestratificada, que se encuentra dominada por árboles altos siempre verdes de 8 a 30 $\mathrm{m}$ de altura. Se pueden distinguir claramente cuatro estratos, el primero se diferencia por árboles altos que van de los 20 a $30 \mathrm{~m}$ de altura y se encuentra representado por Licania platypus Fritsch, Magnolia mexicana DC., C. mollis, Sommera arborescens Schltdl. y $B$. anay. El segundo estrato está conformado por árboles de talla media de entre 6 y $15 \mathrm{~m}$, como los del género Persea. Mientras que el estrato bajo se distingue por la presencia de arbustos con alturas que oscilan entre los 3 y $6 \mathrm{~m}$. Los arbustos más representativos son Celtis iguanaea (Jacq.) Sarg., Piper hispidum Sw., Casearia corymbosa Kunth, T. mexicana y Annona reticulata L. Entre las plantas epífitas dominantes destaca el género Tillandsia y entre las hemiepífitas Syngonium, Philodendron y Anthurium. El estrato herbáceo es dominante, siendo las especies más representativas Chamaedorea pinnatifrons Oerst., Chamaedorea elegans Mart., P. seguine, S. podophyllum y A. flexile, así como otros taxa del grupo Monilophyta.

\section{Estado de conservación de la selva}

Se registró un $75 \%$ de las especies reportadas como estructurales para este tipo de vegetación, con alturas de $30 \mathrm{~m}$ y coberturas de 9 (Tabla 2). El 40\% de las especies registradas en este estudio coincide con las especies reportadas como dominantes en el estrato mayor de la selva alta perennifolia de la región Los Tuxtlas y con $43 \%$ de las reportadas en la selva de Misantla. Entre las especies coincidentes 


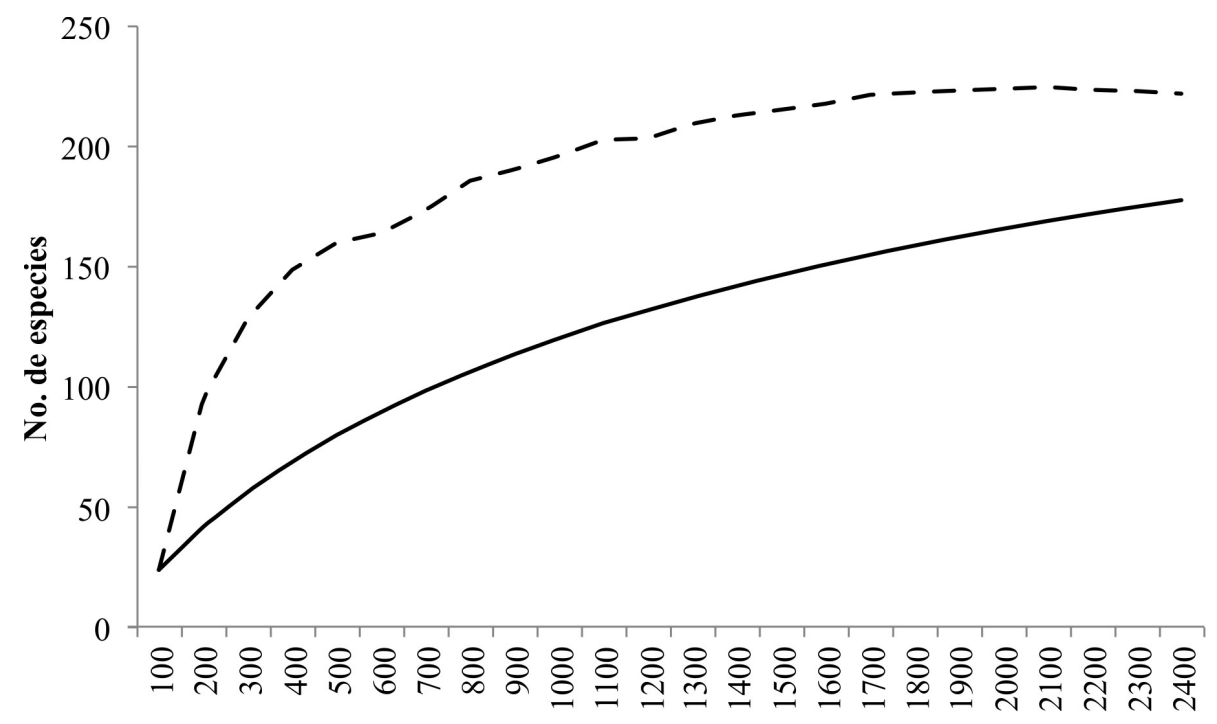

Superficie muestreada en $\mathbf{m}^{2}$

$$
- \text { Sobs } \longrightarrow \text { Chao } 2
$$

Figura 2. Curva de acumulación de especies registradas en remanentes de selva alta perennifolia del centro-norte del estado de Veracruz.

Tabla 1. Riqueza de especies por familias y géneros.

\begin{tabular}{ccccc}
\hline Familias & Especies & Géneros & Géneros & Especies \\
\hline Otras familias & 94 & 82 & Otros géneros & 150 \\
Piperaceae & 15 & 2 & Piper & 9 \\
Araceae & 13 & 5 & Philodendron & 6 \\
Rubiaceae & 11 & 6 & Phychotria & 6 \\
Melastomataceae & 8 & 4 & Peperomia & 5 \\
Malvaceae & 6 & 5 & Miconia & 4 \\
Sapindaceae & 6 & 3 & & \\
Annonaceae & 5 & 4 & & \\
Euphorbiaceae & 5 & 5 & & \\
Moraceae & 5 & 4 & & \\
Urticaceae & 4 & 3 & & 180 \\
Lauraceae & 4 & 4 & & \\
Bromeliaceae & 4 & 2 & & \\
Total & 180 & 129 & &
\end{tabular}

con esta última localidad, podemos mencionar a $B$. anay y Piper schiedeanum Steud. Lo mismo ocurre para el estrato medio o arbustivo, donde se encuentran Alchornea latifolia Sw., Hampea integerrima Schltdl. y M. trinervia. De manera contraria, Pseudolmedia glabrata (Liebm.) C.C.Berg (antes P. oxyphyllaria Donn. Sm.), que es la especie arbórea más abundante en Misantla, fue escasa en la selva de Atzalan.

De acuerdo al análisis de similitud de Jaccard
(Figura 3a), se identifican dos grupos correspondientes a cada sección de selva inventariada, excepto por las parcelas S15 y S16, que mostraron una mayor similitud entre ellas (Jaccard $=0.81$ ), mientras que S13 mostró una evidente disimilitud con el resto de las parcelas. El mismo patrón es observó en el ACP, donde es posible observar marcadas diferencias entre las dos secciones de selva (Figura 3b). La sección B, alberga poblaciones de Persea americana Mill., que no se registran 
Tabla 2. Principales especies que conforman la selva alta perennifolia de Atzalan.

\begin{tabular}{|c|c|c|c|c|c|}
\hline Familia & Especie & NC & $\mathrm{FC}$ & $A(m)$ & $\mathrm{C} / \mathrm{A}$ \\
\hline Euphorbiaceae & Alchornea latifolia Sw. & Vicarilla & A & 30 & 9 \\
\hline Annonaceae & Annona reticulata $\mathrm{L}$. & Platanillo & AR & 1.5 & 2 \\
\hline Annonaceae & Annona glabra L. & Tentepo & AR & 14 & 7 \\
\hline Lauraceae & Beilschmiedia anay (S.F. Blake) Kosterm & Anayo & A & 30 & 9 \\
\hline Icacinaceae & Calatola mollis Standl. & Calatola & A & 25 & 8 \\
\hline Flacourtiaceae & Casearia corymbosa Kunth & Plomillo & AR & 2 & 6 \\
\hline Arecaceae & Chamaedorea elegans Mart. & Tepejilote & $\mathrm{H}$ & 0.6 & 2 \\
\hline Arecaceae & Chamaedorea pinnatifrons Oerst. & Tepejilote & $\mathrm{H}$ & 3.5 & 8 \\
\hline Costaceae & Costus dirzoi García-Mend. \& Ibarra-Manr. & - & $\mathrm{H}$ & 3 & 6 \\
\hline Sapindaceae & Cupania dentata Glaz. & Guacamayo & A & 10 & 7 \\
\hline Annonaceae & Cymbopetalum baillonii R.E.Fr. & Platanillo & A & 25 & 9 \\
\hline Moraceae & Ficus calyculata Mill. & - & A & 20 & 7 \\
\hline Guttiferae & Garcinia intermedia (Pittier) Hammel & Manzanillo & A & 5 & 6 \\
\hline Meliaceae & Guarea glabra Vahl & Azote & $A$ & 4 & 6 \\
\hline Malvaceae & Hampea integerrima Schltdl. & Tecoliste & A & 7 & 7 \\
\hline Magnoliaceae & Magnolia mexicana DC. & Yoloxochitl & A & 30 & 9 \\
\hline Rosaceae & Licania platypus Fritsch & - & A & 3 & 3 \\
\hline Icacinaceae & Oecopetalum mexicanum Greenm. \& C.H.Thomps. & Cachichín & A & 10 & 6 \\
\hline Araceae & Philodendron inaequilaterum Liebm. & Papelillo & B & 15 & 8 \\
\hline Araceae & Philodendron seguine Schott & Papelillo & B & 8 & 8 \\
\hline Piperaceae & Piper amalago L. & Ashte & A & 7 & 7 \\
\hline Moraceae & Pseudolmedia glabrata (Liebm.) C.C.Berg & Tepetomate & A & 7 & 7 \\
\hline Rubiaceae & Palicourea tetragona (Donn.Sm.) C.M.Taylor & Capulín & A & 10 & 6 \\
\hline Moraceae & Trophis mexicana (Liebm.) Bureau & Ramoncillo & A & 7 & 7 \\
\hline Vitaceae & Vitis bourgaeana Planch. & Parra & B & 20 & 8 \\
\hline Rutaceae & Zanthoxylum riedelianum Engl. subsp. Kellermanii (P.Wilson) Reynel & Pisijia & AR & 25 & 9 \\
\hline
\end{tabular}

$\mathrm{NC}$, nombre común; FC, forma de crecimiento $(\mathrm{A}=$ árbol, $\mathrm{AR}=$ arbusto, $\mathrm{H}=$ hierba, $\mathrm{B}=$ bejuco); $\mathrm{A}(\mathrm{m})$, altura máxima en metros; C/A, valor de cobertura abundancia (van deer Marel 1979); - sin dato.

en la sección A y presenta una mayor frecuencia de las principales especies que componen la selva alta perennifolia. Por lo que $B$. anay, típica de vegetación primaria, tuvo su mayor frecuencia en esta área (83\%). Resultados similares se tienen para G. glabra, P. seguine (100\%), P. hispidum $(67 \%)$, P. tetragona $(67 \%)$, M. mexicana $(50 \%)$ y T. mexicana $(75 \%)$.

Se identificaron siete especies incluidas en alguna categoría de riesgo. De acuerdo con la NOM-ECOL-059-2010, Astronium graveolens Jacq., Dieffenbachia seguine Schott, M. mexicana y Sapium macrocarpum Müll. Arg. están catalogadas como amenazadas, mientras que Cyathea schiedeana Domin se registra en riesgo. Dentro de esta última categoría, pero en la Lista Roja, se encuentra Cecropia obtusifolia Bertol., entretanto Cymbopetalum baillonii R. E. Fr. y M. mexicana, están bajo la categoría de vulnerable. Resalta la presencia de Costus dirzoi García-Mend. \& IbarraManr. especie endémica del Estado de Veracruz.

\section{DISCUSIÓN}

\section{Inventario florístico}

Los fragmentos de selva muestreados resguardan una gran riqueza de flora vascular, al registrar 180 especies en un área de $2400 \mathrm{~m}^{2}$. Este número representa el $0.8 \%$ de las plantas vasculares estimadas para el país y el $2.1 \%$ para el Estado de Veracruz (Villaseñor 2016). Las especies registradas equivalen al $37 \%$ de las 485 reportadas por LevyTacher et al. (2006) para la selva Lacandona, al $33 \%$ de las especies registradas en el Monumento Natural Yaxchilán (Meave et al. 2008), al 84\% de las especies registradas en el estado de Tabasco (Vázquez-Negrín et al. 2011), al 37\% de las especies registradas para el Parque Nacional Palenque (Gómez-Domínguez et al. 2015), y al 36\% de las especies registradas en la selva Lacandona (DuránFernández et al. 2016). En lo que respecta al estado de Veracruz, el número de especies inventariado equivale al $20 \%$ de las 949 especies de flora vascular reportadas por Ibarra-Manríquez y Sinaca-Colín 
(a)

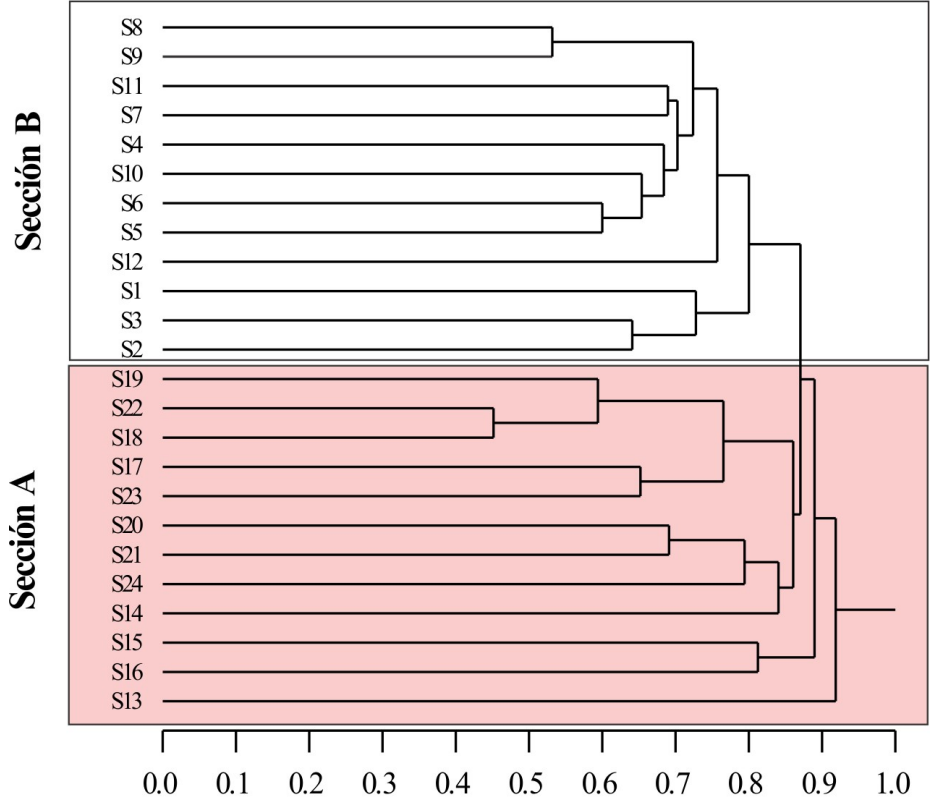

Coeficiente de Jaccard

(b)

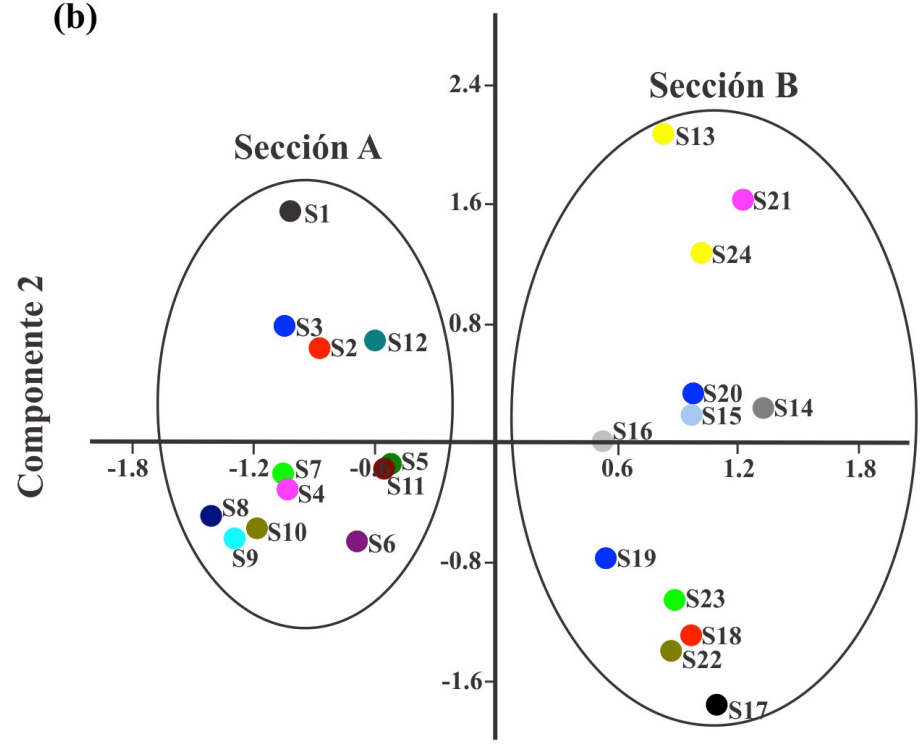

\section{Componente 1}

Figura 3. Variación en la composición florística entre las parcelas estudiadas: a) Dendrograma UPGMA basado en el coeficiente de Jaccard, indicando las similitudes entre parcelas de muestreo, b) Análisis de Componentes Principales (ACP) donde se muestra la separación de las dos secciones de selva. Proyección de los ejes 1 y 2 , que explican el $30 \%$ de la inercia total contenida. S1-S24, parcelas de muestreo. 


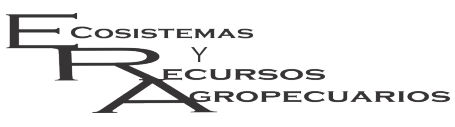

Burgos-Hernández y Castillo-Campos Análisis florístico de la selva tropical Ecosist. Recur. Agropec. 5(15):451-463,2018

(1995) y al $49 \%$ de las especies registradas por Arroyo-Rodríguez et al. (2008). Lo que revela que la selva de Los Tuxtlas constituye uno de los remanentes de este ecosistema en México y remarcan la importancia del fragmento estudiado.

Las familias mejor representadas en la selva de Atzalan, corresponden con las reportadas para selvas altas y medianas perennifolias de México (Arroyo-Rodríguez et al. 2008, Vázquez-Negrín et al. 2011, Gómez-Domínguez et al. 2015). Particularmente Rubiaceae y Euphorbiaceae son dos de las familias con mayor número de taxa (Villaseñor 2016). La primera reúne más de 10000 especies en el mundo, teniendo su máxima expresión en los trópicos (Mabberley 1997). Mientras que la segunda, cuenta con alrededor de 8700 especies, ocupando el sexto lugar en diversidad, después de Orchidaceae, Asteraceae, Fabaceae, Poaceae y Rubiaceae (Steinmann 2002). Por lo que no resulta extraño haber encontrado altamente representadas estas familias. El número de especies registradas para Araceae, representa el $25 \%$ de las especies reportadas para Veracruz (Acebey y Krömer 2008) y el $12 \%$ de las especies reportadas para el país (Croat y Carlsen 2003), por lo que el fragmento estudiado resguarda una considerable riqueza de especies. Los géneros, Piper y Psychotria fueron los más diversos y representativos para esta comunidad vegetal. Ambos, reconocidos por su amplia riqueza de especies tropicales y subtropicales en el país (Villaseñor 2004) y, aunque muchas de ellas han sido consideradas típicas de la vegetación secundaria (Gómez-Pompa 1966, Romero-Romero et al. 2000), también se les puede encontrar en vegetación primaria o poco perturbada (Vázquez-Negrín et al. 2011, Gómez-Domínguez et al. 2015).

\section{Estado de conservación de la selva}

La mayor coincidencia de especies encontrada entre Misantla y Atzalan (43\%) es congruente con su cercanía geográfica, ambas, en el límite más norteño de su distribución. De manera contrastante, la escasa presencia de $P$. glabrata en Atzalan, que en otras selvas es un elemento abundante (VázquezNegrín et al. 2011), puede obedecer a que se encuentra no solo en el límite altitudinal de su distribución, de entre 300 y 700 msnm (GómezPompa 1966), sino también en su límite latitudinal más norteño, por lo que no resulta extraño encontrar mermada su abundancia. Destaca la ausencia de varios géneros característicos de este tipo de vegetación en diversas regiones del país, como Brosimum, Bursera, Dendropanax, Cedrela y Ceiba. De igual manera, ningún miembro de la familia Sapotaceae fue registrado, la cual es típica de la mayoría de las selvas tropicales húmedas en México (Rzedowski 1978).

Para las formas de crecimiento, las herbáceas fueron las más representativas, lo que difiere con el hecho de que las formas arbóreas incluyen el mayor número de especies en la selva tropical (Rzedowski 1978). Cabe destacar que las formas herbáceas registradas en el presente estudio, corresponden en su mayoría, a las especies de Araceae, Arecaceae y al grupo Monilophyta, propias de este tipo de vegetación. En cambio, la ausencia o el escaso número de herbáceas pertenecientes a familias afines a ambientes deteriorados como Fabaceae, Asteraceae y Poaceae, indica un bajo nivel de alteración (Gómez-Pompa y Vázquez-Yáñez 1976). Diferencias en la proporción de formas de crecimiento pueden ser por variación en los factores bióticos y abióticos que se dan de una región a otra (Gentry 1990).

La selva de Atzalan protege siete especies en categoría de riesgo, esta cifra representa el $87.5 \%$ de las especies en riesgo registradas por ArroyoRodríguez et al. (2009) en Los Tuxtlas y el $58 \%$ de las registradas por Levy-Techer et al. (2006) para la selva Lacandona. Especies amenazadas como A. graveolens, M. mexicana, S. macrocarpum y $D$. seguine han sido reportadas también en otras selvas tropicales húmedas del país (Castillo-Campos y Laborde-Dovalí 2004, Levy-Tacher et al. 2006, Arroyo-Rodríguez et al. 2009). La especie Costus dirzoi, fue considerada hasta hace poco como única de Los Tuxtlas (García-Mendoza y IbarraManríquez 1991, Vovides 1994). Recientemente fue registrada en Las Choapas y, en el presente estudio, en poblaciones silvestres en la selva del centro- 
norte del estado, lo que sugiere una mayor distribución geográfica. De acuerdo con Castillo-Campos et al. (2005), las especies endémicas son propias de la vegetación primaria. No obstante, la ampliación de la frontera agrícola y ganadera ha ocasionado que la vegetación original desaparezca a un ritmo acelerado y la riqueza de plantas endémicas se encuentra amenazada (Gómez-Pompa et al. 2010).

El fragmento de selva muestreado, presentó diferencias en la composición de especies entre las dos secciones que lo integran. La porción de selva más cercana al poblado de San Pedro Altepepan (sección B), presenta un mayor porcentaje de especies nativas que la porción más cercana al poblado de La Vega (sección A). Los datos sugieren que la sección $B$ se encuentra en un mejor estado de conservación, lo que coincide con las condiciones socio-ambientales de la zona. La porción de selva perteneciente a esta sección, se encuentra alejada de los asentamientos humanos y carece de vías de acceso, por lo que acceder desde esta comunidad es inviable. Además, esta parte de la selva se caracteriza por presentar un terreno accidentado. En contraste, la sección A, presenta un terreno con menor pendiente y se localiza cercano a la población de La Vega, la cual dispone de mayor accesibilidad a los recursos de la selva.

\section{CONCLUSIONES}

A pesar de la superficie que ocupa el fragmento de selva tropical húmeda en Atzalan, alberga una gran riqueza de especies propias de la vegetación primaria de este ecosistema, incluyendo especies en peligro de extinción, endémicas y de importancia económica. Los datos permiten afirmar que el fragmento de selva muestreado se encuentra aún conservado. Este remanente se encuentra bajo fuerte presión antrópica, de seguir esta tendencia, puede desaparecer. Debido a su pequeña extensión y por desconocimiento de su composición florística, este fragmento ha quedado fuera de las iniciativas de conservación, aun cuando este tipo de remanentes tienen gran valor en la conectividad del paisaje.

\section{AGRADECIMIENTOS}

A los pobladores de las localidades de San Pedro Altepepan, El Quimite y La Vega del Río San Pedro en el municipio de Atzalan por su valiosa colaboración. También a Javier Barrientos Villalobos por su apoyo en la edición de imágenes, a Jerzy Rzedowsky, Carlos Durán, Adriana Hernández Rojas y Leticia Monge González por su ayuda en la identificación de ejemplares botánicos.

\section{LITERATURA CITADA}

Acebey A, Krömer T (2008) Diversidad y distribución de Araceae de la reserva de la biosfera Los Tuxtlas, Veracruz, México. Revista Mexicana de Biodiversidad 2: 466-471.

APG IV (2016) An update of the Angiosperm Phylogeny group classification for the orders and families of flowering plants: APG IV. Botanical Journal of the Linnean Society 18: 1-20.

Arroyo-Rodríguez V, Pineda E, Escobar F, Benítez-Malvido J (2008) Value of small patches in the conservation of plant-species diversity in highly fragmented rainforest. Conservation Biology 23: 729-739.

Arroyo-Rodríguez V, Dunn CJ, Benítez-Malvido J, Mandujano S (2009) Angiosperms, Los Tuxtlas Biosphere Reserve, Veracruz, Mexico. Check List 5: 787-799.

Castillo-Campos G, Laborde-Dovalí J (2004) La vegetación. En: Guevara S, Laborde J, Sánchez-Ríos G (eds.) Los Tuxtlas: el paisaje de la sierra. Instituto de Ecología, Unión Europea. Xalapa, México. pp: 231-265.

Castillo-Campos G, Medina-Abreo M, Dávila-Aranda P, Zavala-Hurtado J (2005) Contribución al conocimiento del endemismo de la flora vascular en Veracruz, México. Acta Botanica Mexicana 73: 19-57. 
Castillo-Campos G, Halffter G, Moreno C (2008) Primary and secondary vegetation patches as contributors to floristic diversity in a tropical deciduous forest landscape. Biodiversity and Conservation 17: 1701-1714.

Challenger A, Dirzo R, López-Acosta JC, Mendoza E, Lira-Noriega A, Cruz I, et al. (2009) Factores de cambio y estado de la biodiversidad. En: Sarukhán J (ed.) Capital natural de México, Vol. 2: Estado de conservación y tendencias de cambio. Comisión Nacional para el Conocimiento y Uso de la Biodiversidad. México. pp: 37-73.

Christenhusz MJM, Zhang XC, Schneider H (2011) A linear sequence of extant families and genera of lycophytes and ferns. Phytotaxa 19: 7-54.

Colwell RK (2013) EstimateS: Statistical estimation of species richness and shared species from samples. Version 9. http//purl.oclc.org/estimates. Fecha de consulta 5 de abril de 2017.

Croat TB, Carlsen M (2003) Araceae. In: Rzedowski J, Calderón de Rzedowski G (eds.) Flora del Bajío y de regiones adyacentes 114. Instituto de Ecología A.C. Pátzcuaro, México. 37p.

Díaz-Gallegos JR, Francois MJ, Velázquez A (2010) Trends of tropical deforestation in Southeast Mexico. Singapore Journal of Tropical Geography 31: 180-196.

Durán-Fernández A, Aguirre-Rivera R, García-Pérez J, Levy-Tacher S, de Nova-Vázquez A (2016) Inventario florístico de la comunidad Lacandona de Nahá, Chiapas, México. Botanical Science 91: 1-28.

Ezcurra E (2016) Anthropogenic disturbances infiltrate forest fragments. Proceedings of the National Academy of Sciences 113: 5150-5152.

Fahrig L (2003) Effects of habitat fragmentation on biodiversity. Annual Review of Ecology, Evolution, and Systematics 34: 487-515.

FRA (2010) Evaluación de los recursos forestales mundiales 2010. Informe Nacional México. Evaluación de los recursos forestales mundiales. Departamento Forestal, Organización de las Naciones Unidas para la Agricultura y la Alimentación. Roma, Italia. 98p.

García-Mendoza A, Ibarra-Manríquez G (1991) A new species of Costus dirzoi (Costoidea, Zingiberaceae) from Veracruz, Mexico. Annals of the Missouri Botanical Garden 78: 1081-1084.

Gentry AH (1990) Four neotropical rainforest. Yale University Press. New Haven, Connecticut, USA. 640p.

Gentry AH (1996) A field guide to the families and genera of woody plants of Northwest South America. University of Chicago Press. Conservation International, Washington, USA. 895p.

Gobierno del Estado de Veracruz (2017) Sistema de información municipal. Cuadernillos municipales: Atzalan. SEFIPLAN Estado de Veracruz, Secretaría de finanzas y planeación. Veracruz, México. 11p.

Gómez-Domínguez H, Pérez FMA, Espinoza JJA, Márquez RMI (2015) Listado florístico del Parque Nacional Palenque. Botanical Science 93: 559-578.

Gómez-Pompa A (1966) Estudios botánicos de la región de Misantla, Veracruz. Instituto Mexicano de Recursos Naturales Renovables. México. 173p.

Gómez-Pompa A, Vázquez-Yáñez C (1976) Estudios sobre sucesión secundaria en los trópicos cálidoshúmedos: el ciclo de vida de las especies secundarias. En: Gómez-Pompa A, Vázquez C, Amo S (eds.) Investigaciones sobre la regeneración de selvas altas en Veracruz, México. CECSA,. México. pp: 579-593. 
Gómez-Pompa A, Krömer T, Castro CR (2010) Atlas de la Flora de Veracruz. Gobierno del Estado de Veracruz. Comisión del Estado de Veracruz para la Conmemoración de la Independencia Nacional y la Revolución Mexicana/Universidad Veracruzana. Xalapa, Veracruz. 526p.

Guevara R, Dirzo R (1998) A rapid method for the assessment of the macromycota. The fungal community of an evergreen cloud forest as an example. Canadian Journal of Botany 76: 596-601.

Guevara S, Dovalí LJ, Sánchez RG (2000) La Reserva de La Biosfera Los Tuxtlas. Documento de trabajo. Programa de cooperación sur-sur sobre desarrollo socioeconómico ambientalmente adecuado en los trópicos húmedos. UNESCO. París, Francia. 49p.

Ibarra-Manríquez G, Sinaca-Colín S (1995) Lista florística comentada de la Estación de Biología Tropical Los Tuxtlas, Veracruz, México. Revista de Biología Tropical 43: 75-115.

IPNI (2017) The International Plant Name Index. http://www.ipni.org/. Fecha de consulta 25 de mayo 2017.

IUCN (2016) The IUCN red list of threatened species versión 2016.2. Red List Unit, Cambridge U.K. http//www.iucnredlist.org. Fecha de consulta 20 septiembre de 2017.

Jiménez-Valverde A, Hortal J (2003) Las curvas de acumulación de especies y la necesidad de evaluar la calidad de los inventarios biológicos. Revista Iberoamericana de Aracnologia 8: 151-161.

Levy-Tacher SI, Aguirre RJ, García JD, Martínez MM (2006) Aspectos florísticos de Lacanhá chansayab, selva Lacandona, Chiapas. Acta Botánica Mexicana 77: 67-98.

López-Gómez M, Williams-Linera G (2006) Evaluación de métodos no paramétricos para la estimación de riqueza de especies de plantas leñosas en cafetales. Boletín de la Sociedad Botánica de México 78: 7-15.

Mabberley DJ (1997) The plant book-a portable dictionary of vascular plants. Cambridge University Press. New York, USA. 874p.

Martínez-Ramos M, Ortiz-Rodríguez IA, Piñero D, Dirzo R, Sarukhán J (2016) Anthropogenic disturbances jeopardize biodiversity conservation within tropical rainforest reserves. Proceedings of the National Academic of Science 19: 5323-5328.

Meave AJ, Romero-Romero MA, Valle-Doménech A, Rincón-Gutiérrez A, Martínez E, Ramos HC (2008) Plant diversity assessment in the Yaxchilán Natural Monument, Chiapas, México. Boletín de la Sociedad Botánica de México 83: 53-76.

Mickel JT, Smith RA (2004) The Pteridophytes of Mexico. Memoirs of the New York Botanical Garden Press. New York, USA. 300p.

Miller K, Chang E, Johnson N (2001) Defining common ground for the Mesoamerican biological corridor. World Resources Institute. Whashington, USA. 45p.

Moreno EC (2001) Métodos para medir la biodiversidad. Manuales y Tesis SEA. Zaragoza, España. 83p.

Oksanen J, Kindt R, Legendre P, O'Hara B, Simpson GL, Solymos P, et al. (2009) Vegan: Community ecology package. R package. http://CRAN.R-project.org/package=vegan. Fecha de consulta 12 de enero de 2017.

Pennington TD, Sarukhán J (2005) Árboles tropicales de México. UNAM. Fondo de Cultura Económica. México. 553p. 
Romero-Romero MA, Castillo S, Meave J, van der Wal H (2000) Análisis florístico de la vegetación secundaria derivada de la selva húmeda de montaña de Santa Cruz Tepetotutla (Oaxaca), México. Boletín de la Sociedad Botánica de México 67: 89-106.

Rzedowski J (1978) Vegetación de México. Limusa. México. 504p.

Rzedowski J (1993) Diversity and origins of the phanerogamic flora of Mexico. In: Ramamoorthy TP, Bye $\mathrm{R}$, Lot A, Fa J (eds.) Biological diversity of Mexico: Origins and distribution. Oxford University Press. New York, USA. pp: 129-144.

Sánchez-Colón S, Flores-Martínez A, Cruz-Leyva IA, Velázquez A (2009) Estado y transformación de los ecosistemas terrestres por causas humanas. En: Sarukhán J (ed.) Capital natural de México, Vol. 2: Estado de conservación y tendencias de cambio. Comisión Nacional para el Conocimiento y Uso de la Biodiversidad. México. pp: 75-129.

Sánchez-Vindas PE (1990) Myrtaceae. En: Gómez-Pompa A, Sosa V (eds.) Flora de Veracruz 62. Instituto de Ecología, A.C.-University of California. Xalapa, México. 146p.

Steinmann VW (2002) Diversidad y endemismo de la familia Euphorbiaceae en México. Acta Botánica Mexicana 61: 61-93.

Systat (2016) SYSTAT 13.1 for windows. San José, California. https://systatsoftware.com/. Fecha de consulta 23 de enero de 2017.

van der Maarel E (1979) Transformation of cover-abundance values in phytosociology and its effects on community similarity. Vegetatio 39: 97-114.

Vázquez-Negrín I, Castillo-Acosta O, Valdez-Hernández IJ, Zavala-Cruz J, Martínez-Sánchez LJ (2011) Estructura y composición florística de la selva alta perennifolia en el Ejido Niños Héroes, Tenosique, Tabasco, México. Polibotánica 32: 41-61.

Villafuerte D, García M, Meza S (1993) Ganaderización y deforestación en el trópico mexicano y sus expresiones en el estado de Chiapas. CINVESTAV-PROAFT. México. 25p.

Villaseñor JL (2004) Los géneros de plantas vasculares de la flora de México. Boletín de la Sociedad Botánica de México 75: 105-135.

Villaseñor JL (2016) Checklist of the native vascular plants af Mexico. Revista Mexicana de Biodiversidad 87: 559-902.

Vovides AP (1994) Costaceae. En: Sosa V (ed.). Flora de Veracruz 78. Instituto Nacional de Investigaciones sobre Recursos Bióticos. Xalapa, México. 20p.

Zambrano J, Coates R, Howe FH (2014) Effects of forest fragmentation on the recruitment success of the tropical tree Poulsenia armata at Los Tuxtlas, Veracruz, Mexico. Journal of Tropical Ecology 30: 209218.

\section{Información complementaria} https://www.researchgate.net/publication/320933919_ANEXO_I 
